\title{
\begin{tabular}{l|l} 
Mibraries & DSpace@MIT
\end{tabular}
}

\author{
MIT Open Access Articles
}

\section{D Excitons as Primary Energy Carriers in Organic Crystals: The Case of Oligoacenes}

The MIT Faculty has made this article openly available. Please share how this access benefits you. Your story matters.

Citation: Emelianova, E.V. et al. "2D Excitons as Primary Energy Carriers in Organic Crystals: The Case of Oligoacenes." Physical Review Letters 104.20 (2010): 206405. @ 2010 The American Physical Society.

As Published: http://dx.doi.org/10.1103/PhysRevLett.104.206405

Publisher: American Physical Society

Persistent URL: http://hdl.handle.net/1721.1/58625

Version: Final published version: final published article, as it appeared in a journal, conference proceedings, or other formally published context

Terms of Use: Article is made available in accordance with the publisher's policy and may be subject to US copyright law. Please refer to the publisher's site for terms of use. 


\title{
2D Excitons as Primary Energy Carriers in Organic Crystals: The Case of Oligoacenes
}

\author{
E. V. Emelianova, ${ }^{1, *}$ S. Athanasopoulos, ${ }^{1}$ R. J. Silbey, ${ }^{2}$ and D. Beljonne ${ }^{1}$ \\ ${ }^{1}$ Laboratory for Chemistry of Novel Materials, University of Mons, Place du Parc 20, B-7000 Mons, Belgium \\ ${ }^{2}$ Massachusetts Institute of Technology, Department of Chemistry and Center for Excitonics, Cambridge, Massachusetts 02139, USA
}

(Received 25 September 2009; published 20 May 2010)

\begin{abstract}
A number of organic crystals show anisotropic excitonic couplings, with weak interlayer interactions between molecules that are more strongly coupled within the layers. The resulting energy carriers are intralayer 2D excitons that diffuse along the interlayer direction. We model this analytically for infinite layers and using quantum-chemical calculations of the electronic couplings for anthracene clusters. We show that the exciton hopping rates and diffusion lengths depend in a subtle manner on the size and shape of the interacting aggregates, temperature, and the presence of energetic disorder.
\end{abstract}

DOI: 10.1103/PhysRevLett.104.206405

Energy transport plays a key role in the working principle of, among others, organic photovoltaic (PV) devices [1]. The limited exciton diffusion length, $L_{D}$, in conjugated polymers [2] is one of the main bottlenecks to the PV efficiencies and has triggered the development of complex architectures based on donor-acceptor bulk heterojunctions. An alternative approach to improve the harvesting of energy carriers in organics is provided by multilayer structures based on well-defined molecular materials. The larger $L_{D}$ values measured for small molecules are usually ascribed to reduced energetic disorder [3-5]. Another key parameter is the magnitude of the excitonic coupling mediating energy transport, which is a subtle function of both the relative molecular arrangements and conjugation length of the interacting chromophores [6].

In a recent work on pentacene- $\mathrm{C}_{60}$ bilayer PV cells, an $L_{D}$ value in excess of $70 \mathrm{~nm}$ has been reported [1]. Pentacene (as well as other polyacenes like anthracene) crystallizes according to a $2 \mathrm{D}$ herringbone structure with much larger excitonic interactions in the $a b$ plane compared to interplane couplings. In [1] the pentacene molecules were evaporated using a layer-by-layer deposition technique, so that $L_{D}$ is likely limited by energy transport along the $c$ axis, i.e., by interlayer (inter- $a b$ plane) exciton motion. Within the framework of Förster energy transfer (ET) theory the small interlayer coupling is apparently at odds with the large $L_{D}$ value.

In this Letter, we develop a general model describing energy transport in polycrystalline organic thin films showing highly anisotropic excitonic interactions, with strong couplings in the layers and weak couplings between the layers. In such structures, the electronic excitations are coupled coherently in the layers forming 2D or "plane wave" exciton states as the primary energy carriers. The energy diffusion dynamics can then be described by hopping of these 2D Frenkel excitons from one layer to the other according to a Förster-like picture [7]. We first develop an analytical model for hopping of $2 \mathrm{D}$ excitons in a model system formed by two infinite layers of molecules (each with one molecule per unit cell). Quantum-chemical
PACS numbers: 71.35.Aa, 82.20.Rp, 78.66.Qn, 66.30.Pa

calculations are then performed: (i) on the model system to analyze the influence of layer topology ( $H$ vs $J$ aggregate), interlayer separation and temperature; and (ii) on finite clusters of a prototypical 2D herringbone organic crystal, anthracene to get a quantitative picture of energy diffusion in organic crystals. In particular, we demonstrate how the interlayer component of the singlet exciton diffusion length can be increased by intralayer delocalization of the excitons, which itself is fixed by the size and shape of the aggregates and the magnitude of diagonal energetic disorder.

For randomly distributed point donor and acceptor dipoles as assumed in Förster theory the ET rate follows a $\left(R_{0} / r\right)^{6}$ distance dependence, where $R_{0}$ is the Förster radius. The applicability of the Förster formula to describe ET in chemical and biological systems has been examined in [7]. In particular, at close donor-acceptor separation, the point dipole approximation breaks down and results in a departure from the $1 / r^{6}$ dependence [8]. If, however, the interlayer separation is large compared to the molecular dimensions (as typically found in oligoacenes), the standard Förster expression for a localized excitation can be easily generalized to one point dipole interacting with a 2D or 3D assembly of point dipoles; the corresponding rate scales as $\left(d_{0} / d\right)^{n}$ with $n=4$ for a sheet of dipoles and $n=$ 3 for a slab, where $d_{0}$ is the critical transport distance [9]. Note, that experimentally, values of $d_{0}$ larger, similar, or even smaller [10-12] to $R_{0}$ have been reported.

The dipole summation approach has been successfully applied to account for the exciton band structures and Davydov splitting in oligoacenes $[13,14]$. Dipole summations in the plane converge quickly $[14,15]$ and, when applied to nonequivalent molecules, provide a quantitative picture of the Davydov splitting in anthracene. However, the first and most important term in the sum corresponds to the electronic coupling between equivalent transition dipoles. Here we apply the two-dimensional dipole sum approach to investigate a model system formed by two infinite layers with one molecule per unit cell and strong (weak) interactions between the molecular transition di- 
poles within (in between) the layers. The electronic coupling between the interlayer $k$-state excitons is:

$$
\begin{aligned}
J_{k k} & =\frac{1}{N} \sum_{p, q} e^{i \vec{k} \cdot(\vec{p}-\vec{q})}\left\langle\Phi_{p, 1} f_{q, 2}|V| f_{p, 1} \Phi_{q, 2}\right\rangle \\
& =\frac{1}{N} \sum_{l, p} e^{i \vec{k} \cdot \vec{l}}\left\langle\Phi_{p, 1} f_{p-l, 2}|V| f_{p, 1} \Phi_{p-l, 2}\right\rangle,
\end{aligned}
$$

with $\Phi_{p, 1}$ the excited molecular wavefunction on site $p$ in layer $1, \vec{l}=\vec{p}-\vec{q}$ and $f_{p, 1}$ the ground state molecular wave function at site $p$ in layer 1 (see Fig. 1).

For dipoles parallel to each other and perpendicular to the molecular layers, i.e., for molecules adopting $H$ aggregate-like configurations [see Fig. 1(a)], the electronic coupling Eq. (1) takes the form: $J_{k k}=$ $\left(\mu^{2} / d^{3}\right) \sum_{l} e^{i \vec{k} \cdot \vec{l}}\left\{\left[(l / d)^{2}+1\right]^{-3 / 2}-3\left[(l / d)^{2}+1\right]^{-5 / 2}\right\}$, where $d$ is the distance between the (infinite) layers and $\mu$ is the molecular transition dipole moment. In the continuum approximation, one can write the electronic coupling $J_{k k}$ and the sums $\sum_{k} J_{k k}, \sum_{k} J_{k k}^{2}$ as:

$$
\begin{gathered}
J_{k k}=-\left(\mu^{2} / A\right) k e^{-k d}, \quad \sum_{k} J_{k k}=-2 N\left(\mu^{2} / d^{3}\right), \\
\sum_{k} J_{k k}^{2}=(3 \pi / 4) N\left(\mu^{4} /\left[A d^{4}\right]\right),
\end{gathered}
$$

where $A$ is the unit cell area. Assuming a simple Gaussianlike shape for the exciton absorption and emission spectra [16], the Förster rate for exciton hopping reads:

$$
k_{F}=(2 \pi / \hbar) \sum_{k, k^{\prime}} P_{k} J_{k k^{\prime}}^{2} F_{k k^{\prime}},
$$

where $\quad F_{k k^{\prime}}=\left(1 / \sqrt{2 \pi} \sigma_{h}\right) \exp \left[-\left(E_{k}-E_{k^{\prime}}-\Delta_{\mathrm{Ss}}\right)^{2} /\left(2 \sigma_{h}^{2}\right)\right]$ defines the spectral overlap between $k$-state emission and $k^{\prime}$-state absorption, $\Delta_{\mathrm{Ss}}$ is the Stokes shift and $P_{k}$ the Boltzmann weighted population of $k$ states of the donor and $\sigma_{h}=\sqrt{2 k_{B} T \Delta_{\mathrm{Ss}}}$. In the case of narrow excitonic band or in the high temperature regime $\left|E_{k=0}-E_{k=(\pi / A)}\right| \ll$ $k_{B} T$, the excitonic states are equally populated with $P_{k} \cong$ $(1 / N)$. In this limit $k_{F} \cong \frac{3 \pi}{4} \frac{\sqrt{2 \pi}}{\hbar} \frac{1}{\sigma_{h}} \exp \left[-\frac{\Delta_{\mathrm{ss}}}{4 k_{B} T}\right] \frac{\mu^{4}}{A d^{4}}$ and the value of the critical transfer distance is $d_{0}=\mu\left(\frac{\tau}{A \hbar \sigma_{h}}\right)^{1 / 4} \times$ $\left[\frac{3 \pi^{2}}{2 \sqrt{2 \pi}} \exp \left(-\frac{\Delta_{\mathrm{Ss}}}{4 k_{B} T}\right)\right]^{1 / 4}$, with $\tau$ the radiative decay time.

Similarly, if the dipoles are lying parallel to each other and in plane [Fig. 1(b)], i.e., for layers displaying $J$
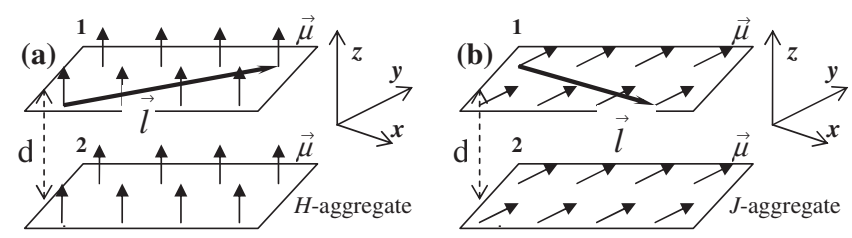

FIG. 1. Model system: two identical, infinite donor and acceptor layers, each with one molecule per unit cell. (a) The molecular transition dipoles are parallel to the $z$ axis; (b) the molecular transition dipoles are lying in the $x y$ plane. aggregate-like configurations, we obtain (taking for simplicity the direction of the $x$ axis along $\vec{k}$ ):

$$
\begin{gathered}
J_{k k}=(1 / A)(\vec{k} \cdot \vec{\mu})^{2} e^{-k d} / k, \quad \sum_{k} J_{k k}=N\left(\mu^{2} / d^{3}\right), \\
\sum_{k} J_{k k}^{2}=(9 \pi / 32) N\left(\mu^{4} /\left[A d^{4}\right]\right) .
\end{gathered}
$$

For the limiting case of narrow exciton band and/or high temperature, $\quad k_{F} \cong \frac{9 \pi}{32} \frac{\sqrt{2 \pi}}{\hbar} \frac{1}{\sigma_{h}} \exp \left[-\frac{\Delta_{\mathrm{Ss}}}{4 k_{B} T}\right] \frac{\mu^{4}}{A d^{4}}, \quad d_{0}=$ $\mu\left(\frac{\tau}{A \hbar \sigma_{h}}\right)^{1 / 4}\left[\frac{9 \pi^{2}}{16} \frac{1}{\sqrt{2 \pi}} \exp \left(-\frac{\Delta_{\mathrm{Ss}}}{4 k_{B} T}\right)\right]^{1 / 4}$. A few interesting features emerge from the results obtained for the infinite layers: (i) In both $H$ - and $J$-like configurations, a $1 / d^{4}$ distance dependence is predicted for the hopping rate in the high-temperature-narrow-band regime. We should emphasize that in the case of equal populations among the 2D exciton states, identical results are obtained by assuming that the energy carriers are localized on molecular sites. Thus, the weaker decrease of the interlayer rate with distance compared to that predicted by Förster reflects the 2D delocalization of the exciton and/or the dimensionality of the acceptor aggregate [11]. (ii) For an arrangement of the dipoles in a $J$ aggregate, the $k=0$ state at the bottom of the exciton band that concentrates the entire oscillator strength is inactive in the ET process. The same conclusions apply for $1 \mathrm{D}$ molecular wires such as conjugated polymers. This particular feature in conjunction with the large band width promoted by through-bond interactions along the chains results in vanishingly small interchain excitonic coupling in polymers with extended conjugation [17]. (iii) The two types of arrangements yield different $k$-dependent contributions to the overall hopping rate: in both types of aggregates, $J_{k k}^{2}\left(E_{k}\right)$ peaks in the middle of the band when the interlayer distance is comparable with the lattice spacing within the layer. However, for (larger) interlayer distances that are in line with anisotropic excitonic interactions in most organic crystals, the maximum occurs near the top of the exciton band for $H$ aggregates, yet close to the bottom of the band for $J$ aggregates. Those different behaviors of $J_{k k}^{2}\left(E_{k}\right)$ are reflected in the temperature dependence of the hopping rate as only the states near the bottom of the energy band are populated at low $T$. We should emphasize that the model can be generalized to the case of two infinite layers with two molecules per unit cell. Dipole summation applied to nonequivalent molecules have the same form as Eqs. (2) and (4) and therefore do not change the results discussed above [18].

To quantify the above dependencies, we have performed calculations on two model rectangular crystal layers each consisting of $N=121$ anthracene molecules (one molecule per unit cell) with transition dipoles either perpendicular $(H)$ or parallel $(J)$ to the plane of the layer. In what follows we have calculated the excitonic coupling $V_{i j}$ for each anthracene molecule $i$ in the plane and all of the intraand interplane neighboring molecules $j$ by applying periodic boundary conditions and using the distributed monopole approximation [19] that expresses $V_{i j}$ as a Coulomb 
interaction of atomic transition charge densities (calculated by combining the INDO Hamiltonian to a coupled cluster single and double, CCSD, formalism [20]). Transfer rates $k_{F}(T)$ were computed on the basis of Eq. (3) with a spectral overlap fixed to a $T$-independent value (in order to disentangle electronic and vibrational effects). The results are presented in Fig. 2 where all curves have been normalized to the ET rate value calculated in the case of equally populated excitonic states.

Although the overall $T$ dependence will be affected by the coupling to the bath and the resulting homogeneous linewidth, Fig. 2 reveals a very strong asymmetry in $k_{F}(T)$ between the two geometric configurations: the monotonic increase with $T$ of $k_{F}$ in $H$ aggregates arises from population of higher-lying $k$ exciton states with significant $J_{k k}$ values. The same evolution is found for $J$ aggregates at interlayer separations that are commensurate with the interlayer unit lattice parameters. At larger interlayer distances, however, the relative ET rate features a nonmonotonic evolution with a peak at intermediate temperatures. This can be rationalized by the shift towards lower $k$ values at which $J_{k k}$ peaks as the interlayer distance increases. Hence, the higher population of the low-energy exciton states at lower temperatures promotes more efficient energy pathways and translates into higher ET rates.

We now turn our attention to simulations on the actual anthracene single crystal structure [21] that include both $J$ and $H$-like interactions. The INDO-CCSD excitonic couplings yield a Davydov splitting between the lowest $x$-polarized and $y$-polarized absorption bands of $\sim 200 \mathrm{~cm}^{-1}$, in good agreement with the experimental value in the single crystal [15]. However, it is very likely that the electronic excitations in organic thin films are confined in space due to both extrinsic (chemical and structural defects) and intrinsic (coupling to phonons) factors. We have thus computed the rates between two identical anthracene layers as a function of the number of molecules within the clusters, see Fig. 3. $k_{F}$ is found to increase with aggregate size and saturates after a few hundreds of molecules. This dependence is the result of a subtle interplay between thermal population of the initial exciton states, the number of molecules coherently coupled in plane and the distance between the layers. The rate would be vanishingly small for infinite layers, if only the lowest, bright exciton state (see Fig. 3, open circles) would contribute (as is the case in conjugated polymers with large intrachain electronic bands, vide supra).

It is also interesting to explore the distance-dependent exciton hopping rates for layers of increasing size (see inset of Fig. 3). $k_{F}$ between two anthracene molecules reflects the expected $1 / d^{6}$ dependence for point to-point interactions while the exponent fitted to the distancedependent curve converges toward 3-3.5 in the 2D crystal limit. The deviation from the $1 / d^{4}$ law $[9,22]$ can be explained by the fact that low-lying optically forbidden states provide significant contributions to the hopping rate, as in the model $J$ aggregates above.

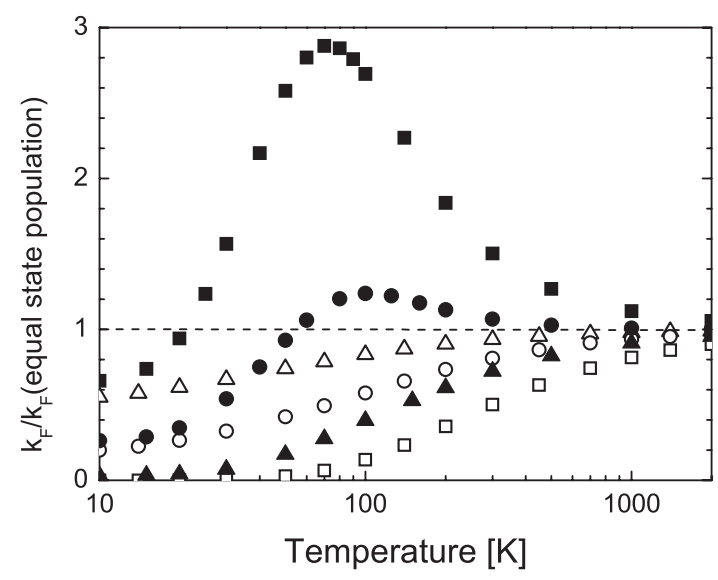

FIG. 2. Temperature dependence of the normalized ET rate for a model system of two rectangular crystal layers with $N=121$ anthracene molecules, for interlayer distances $d$ (see Fig. 1) in $\AA$ :

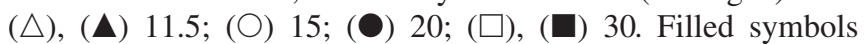
correspond to $J$ aggregate configuration with intermolecular distance $8 \AA$; open symbols correspond to $H$ aggregate configuration with intermolecular distance $12 \AA$.

To gauge the influence of exciton delocalization on transport properties, we have estimated the diffusion coefficient $D$ and the diffusion length based on a "randomwalk" model: $L_{D}=\sqrt{D \tau}=d \sqrt{k_{F} \tau}$. The value of $\tau$ strongly depends upon the nature and density of structural defects in the crystal; therefore, we use here the lower and upper bounds for anthracene thin films of 1 and $4 \mathrm{~ns}$, respectively $[21,23]$. The theoretical values for $L_{D}$ converge to $\sim 100 \mathrm{~nm}$ in the infinite layers for $\tau=1 \mathrm{~ns}$ and $\sim 200 \mathrm{~nm}$ for $\tau=4 \mathrm{~ns}$ (see Fig. 4). These are significantly larger than the values reported in the literature for oligoacene thin films (e.g., $70 \mathrm{~nm}$ in pentacene [1]), which is partly due to disorder effects neglected so far in the calculations.

The influence of uncorrelated energetic (diagonal) disorder on the ET process is illustrated in Fig. 4 (inset), which shows the average (over 500 disorder realizations) $L_{D}$ as a function of the standard deviation $\sigma$ of the Gaussian energy distribution. As expected, disorder significantly reduces $L_{D}$ with typical values for $\sigma$ in molecular crystals $(0.05-0.06 \mathrm{eV})$ resulting in diffusion lengths that are 2-3 times smaller than in disorder-free molecular crystals and in the range of $65-110 \mathrm{~nm}$.

In oligoacenes, the primary photoexcitations can be thought of as 2D excitons delocalized in plane, which act as the primary energy carriers for interlayer hopping motion. We have developed a simple model that, in the limiting case of defect free periodic structures, accounts for the salient features of such a transport regime and where the diffusion length is formulated analytically. In particular, for $J$ aggregates, in case of very large intralayer interactions or very low temperature, only the bottom $k$ exciton states contribute to the ET rate and result in very small $k_{F}$ values. For intralayer couplings that are much 


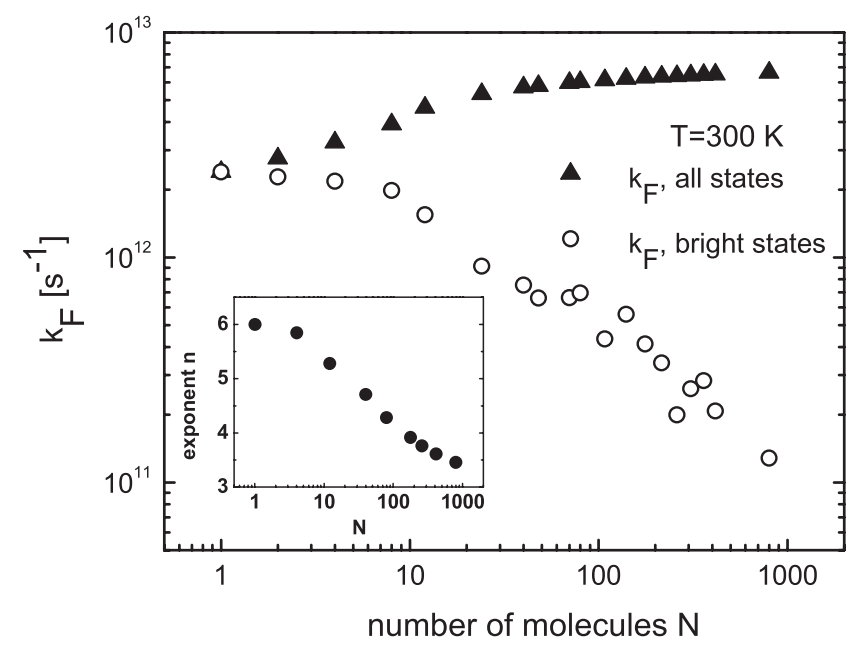

FIG. 3. ( $\boldsymbol{\Delta})$ ET rate between anthracene layers vs the number of molecules within the layers, $(\bigcirc) k_{F}$ calculated from the bright states only. The inset displays the exponent $n$ (obtained from a $(1 / d)^{n}$ fit of $k_{F}$ ) as a function of the anthracene layer size.

larger than their interlayer counterparts but yield exciton band widths commensurate with $k_{B} T$ (as typically found in oligoacenes in the range $100-300 \mathrm{~K}), k_{F}$ is significantly enhanced compared to the single molecule hopping rate as a result of many contributing channels involving optically dark exciton states. We propose that, while the first scenario applies to conjugated polymers (that are a 1D analogue of intralayer 2D excitons with large band widths), small molecules adopting herringbone like structural arrangements such as oligoacenes thin films and crystals pertain to the second regime, thereby rationalizing the very different $L_{D}$ measured in the two types of materials. Quantum-chemical calculations in anthracene layers show a much weaker distance dependence of $k_{F}$ than that predicted from simple Förster hopping and $L_{D}$ values in excess of a few hundreds nm (brought down closer to the

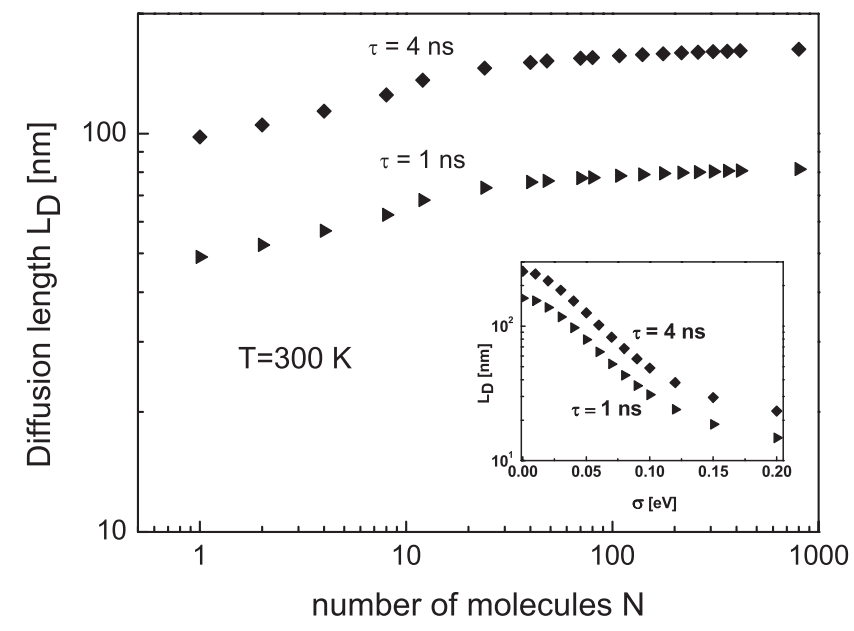

FIG. 4. Exciton $L_{D}$ vs anthracene layer size for exciton lifetimes $\tau$ : $(\downarrow) 1 \mathrm{~ns},(\diamond) 4 \mathrm{~ns}$. The inset shows $L_{D}$ as a function of the standard deviation $\sigma$ of the Gaussian energy distribution. measured $L_{D}$ of $\sim 70 \mathrm{~nm}$ when accounting for diagonal energetic disorder). The method is general and can be applied to other oligoacene molecular crystals that display anisotropic couplings within and in between layers, given a correct description of the excited states at the quantum chemical level. For example, a more elaborate task would be to apply the model in pentacene where the charge transfer configurations contribute significantly to the lowest excited state [24]. Including such effects would essentially lead to a renormalization of the excitonic couplings.

The work in Mons is partly supported by IAP $6 / 27$ of the Belgian Federal Government, the EU projects MODECOM (NMP3-CT-2006-016434) and ONE-P (NMP3-LA-2008-212311), FNRS and FRFC through the Interuniversity Scientific Calculation Facility, ISCF. E. E. thanks V. Geskin for useful discussions. R. J. S. was supported as part of the Center for Excitonics, an Energy Frontier Research Center funded by U.S. Department of Energy, Office of Science, Office of Basic Energy Science and Grant No DE-SC0001088.

*evguenia@averell.umh.ac.be

[1] S. Yoo et al., Solid State Electron. 51, 1367 (2007).

[2] O. V. Mikhnenko et al., J. Phys. Chem. B 112, 11601 (2008).

[3] A. Huijser et al., J. Am. Chem. Soc. 130, 12496 (2008).

[4] A. Mani, J. Schoonman, and A. Goossens, J. Phys. Chem. B 109, 4829 (2005).

[5] S. Athanasopoulos et al., Phys. Rev. B 80, 195209 (2009).

[6] J. Gierschner et al., J. Chem. Phys. 130, 044105 (2009).

[7] D. Beljonne et al., J. Phys. Chem. B 113, 6583 (2009).

[8] Y. R. Khan, T. E. Dykstra, and G. D. Scholes, Chem. Phys. Lett. 461, 305 (2008).

[9] H. Kuhn, J. Chem. Phys. 53, 101 (1970).

[10] J. Hill et al., Phys. Rev. B 69, 041303 (2004).

[11] S. R. Scully et al., Adv. Mater. 19, 2961 (2007).

[12] P. E. Shaw, A. Ruseckas, and I. D. W. Samuel, Adv. Mater. 20, 3516 (2008).

[13] R. Silbey, J. Jortner, and S. A. Rice, J. Chem. Phys. 42, 1515 (1965).

[14] M. R. Philpott, J. Chem. Phys. 59, 4406 (1973).

[15] M. R. Philpott, J. Chem. Phys. 58, 588 (1973).

[16] T. S. Ahn, N. Wright, and C. J. Bardeen, Chem. Phys. Lett. 446, 43 (2007).

[17] D. Beljonne et al., J. Chem. Phys. 112, 4749 (2000).

[18] The Davydov splitting value can be estimated as the double sum of dipole-dipole interaction term between nonequivalent molecules (e.g., [13]) and takes the form $2 J_{k=0}=\left(2 \mu^{2} / A r_{N}\right) \cos (\varphi)$, where $r_{N}$ is the distance between nonequivalent molecules in the plane and $\varphi$ the angle between their transition dipole moments.

[19] E. Hennebicq et al., J. Am. Chem. Soc. 127, 4744 (2005).

[20] Z. Shuai and J. L. Brédas, Phys. Rev. B 62, 15452 (2000).

[21] T. S. Ahn et al., J. Chem. Phys. 128, 054505 (2008).

[22] P. E. Shaw, A. Ruseckas, and I. D. W. Samuel, Phys. Rev. B 78, 245201 (2008).

[23] R. C. Powell and Z. G. Soos, J. Lumin. 11, 1 (1975).

[24] D. Beljonne et al. (to be published). 